\title{
Perspectives of surrogate decision makers for critically ill patients regarding gene variation research
}

\author{
Ellen Iverson, $\mathrm{MPH}^{1}$, Aaron Celious, $\mathrm{PhD}^{1}$, Carie R. Kennedy, RN² , Erica Shehane, $\mathrm{MPH}^{1}$, \\ Alexander Eastman, MD, $\mathrm{MPH}^{3}$, Victoria Warren, $\mathrm{RN}^{3}$ and Bradley D. Freeman, $\mathrm{MD}^{2}$
}

Purpose: We undertook this investigation to explore the manner in which surrogate decision makers for critically ill patients perceived genetic data collected in the context of clinical investigation.

Methods: Surrogate decision makers for critically ill patients cared for in intensive care units of two urban hospitals participated in focus groups designed to explore perceptions regarding gene variation research.

Results: Surrogate decision makers were generally familiar with genetic concepts and reported that they could provide an informed opinion regarding permitting (or declining) the participation of their loved ones in gene variation research. Respondents perceived the risk associated with this type of research largely as the risk associated with acquiring the sample (i.e., whether it involved an invasive procedure or not) but appreciated that genetic samples could provide information not readily obtained from nongenetic sources. Concerns

\section{INTRODUCTION}

Genetic variation influences disease profile and treatment response. ${ }^{1}$ Gene-based technologies may prove an important adjunct to conventional approaches to providing care and have applications in most spheres of clinical practice, including management of critically ill patients. ${ }^{1,2}$ Recent advances have produced a more detailed understanding of genetic architecture and have facilitated acquisition and analysis of genetic data in an economical fashion. ${ }^{3,4}$ One consequence of these developments is that genetic data are commonly collected as a facet of epidemiological and intervention studies, including those conducted in acutely ill individuals. ${ }^{2,5}$

Critical illness constitutes a substantial disease burden, and investigations enrolling acutely ill patients are essential to therapeutic advances. ${ }^{6}$ Clinical studies conducted in this context pose unique hurdles. ${ }^{6}$ Conditions resulting in admission to intensive care units (ICUs) are often sudden in onset, and the care provided is complex. ${ }^{6}$ Patients are commonly incapacitated as a result of their disease or treatment..$^{6-9}$ Enrollment in critical illness studies requires that permission be obtained expediently from surrogate decision makers (SDMs) (e.g., family members, guardians, or domestic partners). ${ }^{6}$

Investigations involving cognitively impaired individuals are perceived as carrying greater risk and requiring additional safeguards as compared with studies enrolling those about potential misuse of genetic data largely centered on misconduct, paternity, forensic applications, and insurance and employment discrimination. Although surrogate decision makers expressed that their loved ones would have interest in return of results and being recontacted for future use, these interests were secondary to confidentiality concerns.

Conclusion: Respondents perceived genetic and nongenetic data as comparable. Informed consent processes that provide clear information regarding confidentiality protections, specimen handling, and parameters for future use may enhance enrollment.

Genet Med 2013:15(5):368-373

Key Words: critical illness; focus groups; gene variation research; genetics; social science research; substituted judgment; surrogate decision maker

who are cognitively intact. ${ }^{10}$ When these studies entail collection of genetic material, such concerns appear heightened. ${ }^{10,11}$ Although a number of investigations have examined attitudes regarding gene variation research, the manner in which the exigencies of acute illness affect perceptions of genetic data collection is largely unknown. ${ }^{12-30}$ Conceivably, SDMs do not possess sufficient insight as to the nature of genetic data to permit informed judgment. Alternatively, the emotional distress that SDMs experience potentially overwhelms concerns that genetic data may possess characteristics that render them distinct from other commonly collected types of information. ${ }^{31-33}$ Given the renewed interest in understanding stakeholder preferences as a foundation for the ethical conduct of genetic research, filling this knowledge gap is essential. ${ }^{34}$

\section{MATERIALS AND METHODS}

\section{SDM sample and recruitment}

Our methodology has been described previously. ${ }^{35}$ Participants were recruited from the surgical and medical ICUs of two tertiary-care institutions (Barnes Jewish Hospital (BJH), St. Louis, MO and Parkland Hospital (PH), Dallas, TX). These study sites were chosen because of their demographic and ethnic diversity. Eligible participants were SDMs for adult patients in the ICUs (age $\geq 18$ years) who were intubated and mechanically ventilated for $\geq 48 \mathrm{~h}$, and who were expected to require ventilatory support

${ }^{1}$ Department of Pediatrics, Children's Hospital Los Angeles, Los Angeles, California, USA; ${ }^{2}$ Department of Surgery, Washington University School of Medicine, St. Louis, Missouri, USA; ${ }^{3}$ Department of Surgery, University of Texas Southwestern Medical Center, Dallas, Texas, USA. Correspondence: Bradley D. Freeman (freemanb@wustl.edu) 
for $\geq 24$ additional hours. Patients who were either incarcerated or pregnant were excluded. Eligible SDMs were approached by research staff and invited to participate; those agreeing provided informed consent and received a $\$ 50$ honorarium.

\section{Data collection}

Data collection (February 2008-August 2009) occurred during eight, 2-wk periods, which were scheduled approximately every 3-4 months to allow for complete patient turnover in participating ICUs. We were interested in obtaining the perspectives of individuals actively engaged in decision making for critically ill patients. Accordingly, focus groups (FGs) were conducted in conference rooms adjoining the ICUs while the loved ones of SDMs received treatment for their critical illness. FG study guides were developed on the basis of themes related to gene variation research, including general familiarity with genetic data, ${ }^{23}$ features that might render genetic data distinct from nongenetic data, ${ }^{13}$ concerns about misuse, ${ }^{12,23}$ confidentiality, ${ }^{18,23}$ and future use. ${ }^{17}$ Hypothetical cases, vignettes, and samples of the institutional review board (IRB)-approved consent language were used to generate discussion. In addition, FG moderators presented scenarios with which participants might have familiarity (e.g., serum cholesterol testing) to illustrate key concepts. Of note, the emphasis of these sessions was on participants serving as SDMs and thus making decisions for patients in a research context. Twenty FGs were conducted (11 at BJH, 9 at $\mathrm{PH}$ ), enrolling 74 SDMs. Participants (mean $( \pm$ SE) age $48( \pm 1.7)$ years) were English-speaking, predominately female (73\%), and described long-term relationships with their loved ones (33 $( \pm 1.5)$ years). Two facilitators (A.C., E.S.) moderated all FG discussions, and a third investigator (E.I.) provided additional notation. Facilitators served either as a discussion leader or tracked speaker identity during the session by using a split microphone and indicating the participants' study code into the microphone as they spoke. This allowed the transcriber to label each narrative segment with these participant codes and enabled linking remarks made by the same individuals throughout the FG session.

\section{Analysis}

Analysis of FG transcripts was based on grounded theory, an iterative process of simultaneous data collection, analysis, and theory construction. ${ }^{36}$ Analysis was facilitated using qualitative analytical software (Atlas.ti, Berlin), which makes it possible to categorize quotes within transcripts and to code themes and analyze relationships between and within text segments. This process begins with the construction of codes that represent themes addressed in the study guide. Findings reported here are based on analysis of narratives corresponding to the following codes: benefits of genetic research/genetic testing, DNA, data access, decisions regarding genetic research, exceptionalism, experience with genetics, meaning of genes/genetic test, negative attributes of genetics, questions regarding genetics, and secondary use of genetic information.

\section{Protection of human subjects}

This study was approved by the Human Studies Committees of Washington University School of Medicine and University of Texas Southwestern Medical Center (HRPO 06-0637).

\section{RESULTS}

\section{Familiarity with genetic concepts, stress of SDM role}

Although most participants possessed at least superficial understanding of genetic concepts that they had acquired via formal education, reading, online resources, and television, several individuals displayed more in-depth knowledge as a result of discussion with acquaintances who had been tested for familial forms of breast and colon cancer. In addition, one individual was familiar with Web-based, direct-to-consumer genetic testing services, such as those marketed to assess disease predisposition and ancestry. We probed how the emotional duress of serving as an SDM might interfere with accurately processing information pertaining to genetic data collection. Although many respondents described their situation as "stressful," they nonetheless felt capable of making informed decisions regarding genetic data collection. However, a minority of FG participants felt that it would be difficult to make such judgments. One individual stated, "I don't know if those questions would ever enter my mind. In a critical situation, to stop and think"so wait a minute, where did they put that sample? Where's it going? Who's going to touch it? Where will they store it?"-That would never even enter my mind. Those things would enter my mind after the fact when you had time to consciously and logically think about it."

\section{Are genetic data unique?}

We explored whether respondents distinguished between genetic and nongenetic data. Many respondents appeared to categorize data on the basis of whether an invasive procedure was necessary to acquire it.

$<$ Respondent $>$ "I would want to know if she is going to have an invasive procedure. You don't have to call and tell me that we're going to do a blood test or a genetic test. That's something that you should be able to do automatically because it's not like she's in extreme risk by you doing that. I mean it's not like she has to have some anesthesia and something could go wrong and she could die."

Many FG participants echoed this view, stating that they would agree to collection of genetic data "as long as it's not invasive." Several participants stated that it would be acceptable to collect such information without specific informed consent.

$<$ Moderator> "Would it be okay if they just said "we're gonna give it [the genetic sample] to the researchers", without giving you the option to choose?

$<$ Respondent> "I think I'd be okay with it. They [the researchers] know the best and they know what they're doing, we don't." 


\section{ORIGINAL RESEARCH ARTICLE}

$<$ Moderator> "So you wouldn't need to have the option, you wouldn't care if it was on a consent form?"

$<$ Respondent> "No, I wouldn't."

Other individuals viewed genetic data as being more informative than nongenetic data, for instance, by providing information about ancestry or disease predisposition, or being used to establish paternity or for forensic purposes. Some noted the distinction that factors assessable by a routine test, such as serum cholesterol level, could be modified through lifestyle changes or medications, whereas those assessable by a genetic test would be less mutable.

$<$ Moderator $>$ "How would you compare a genetic test with a cholesterol test?"

$<$ Respondent> "I look at them differently because you can't control a genetic test. With cholesterol, you can change your diet, you can exercise more, you can take drugs. There's not a whole lot you could do to change your genes. I mean if you got them, that's what it is."

In summary, FG participants perceived that genetic samples could provide information distinct from that obtained from other types of clinical data.

\section{Potential misuse}

We explored concerns about potential for misuse of genetic information. One set of concerns expressed by several participants was related to the use of genetic data to establish paternity. "If a person thought that (these individuals) were their mom and dad, and then they realize that (these individuals) weren't their mom and dad, that would be a negative situation." Similarly, concern was expressed for the potential of genetic data to be used for forensic applications.

$<$ Moderator $>$ "What type of concerns would you have if you were asked to give your sample of DNA?"

$<$ Respondent> "My concern would be, OK, is there anybody saying I'm the father of some kids? Did somebody commit a crime? There's a lot of things they could use your DNA for, and I would be very skeptical about what they're going to use it for."

Respondents also expressed concern that identifying predisposition to diseases that the respondents had not previously considered would be anxiety producing.

$<$ Respondent $>$ "[Identifying diseases] that run in our family, of course it would help me keep healthy. But then on the flip side is that I don't want to be put on medication or treated, or have a lot of doctor's visits just because you trace back and saw that there were diseases in my family, they could skip me."

Finally, respondents voiced concern regarding the potential for genetic data to be used for insurance and employment discrimination.
$<$ Respondent> "Don't you think that a lot of people fear genetic testing because of insurance companies finding out what might be coming up? That would be my biggest fear." $<$ Respondent $>$ "I think from an insurance standpoint, they could deny your coverage because of what you may come into later on in life. My greatest fear is that it would be used against you, and you wouldn't be able to have any coverage."

Some respondents similarly expressed concern that companies might "start picking and choosing people who would be healthier" or select employees on the basis of "certain genetic tendencies" or practices considered "very discriminatory." Respondents reported that these opinions regarding potential misuse of genetic data were not only their own, but that they accurately reflected the perspectives of their critically ill loved ones and would influence decision making with respect to permitting sample collection.

Finally, respondents expressed concerns about genetic samples being used for purposes other than those originally intended and sought assurance that samples would be "used for medical reasons only" and "not be available to employers" and "not get into the wrong medical hands for the wrong types of research," specifically "cloning" and "eugenic" applications. Many participants wanted to know the precise uses for which the data was being collected. "It's not that we're against it at all, we want to know exactly why. What is it that you want it for? What are you going to do with it and how is it going to help?"

\section{Confidentiality concerns}

Respondents voiced a preference for genetic data to be collected in an anonymous fashion. For the purposes of these discussions, anonymous samples were defined as samples devoid of all identifying information.

$<$ Moderator $>$ "So would it ease your anxiety about your loved one participation if the sample was completely anonymous?"

$<$ Respondent $>$ "Oh, definitely. But I wonder if that would even be possible because he is in the hospital and his name is all over everything. If it were possible, to anonymously collect the sample, I think that would be fine."

$<$ Moderator $>$ "Who would you not feel comfortable getting ahold of the results of your loved one's genetic tests?"

$<$ Respondent $>$ "Federal government. Insurance companies. Those sorts of things. Anybody else besides the researchers."

Some respondents stated that they assumed that data collected in a hospital setting would be maintained in a confidential fashion. "Most people assume in a hospital environment, records are kept confidential ... especially, blood testing [results] are kept pretty confidential."

When directly questioned, there did not appear to be a distinction between genetic and nongenetic tests with respect to confidentiality concerns. 
$<$ Moderator> "Are the confidentiality concerns the same for a routine blood test and a genetic sample?"

$<$ Respondent> "Yeah."

Finally, participants stated that their loved ones would be interested in learning the results of clinical studies in which genetic data were collected. However, when it was explained that return of results would require that specimens be identified so as to enable contact, interest in return of results diminished. There was a strong bias toward collection of data in an anonymous fashion.

\section{Future use}

Most respondents were receptive to future use of samples but expressed an interest in some form of recontact with either the person providing consent or with their loved one regarding such studies, specifically to understand what the specimen would be used for and who would have access to it.

$<$ Moderator> "Assume that you've provided permission for your loved ones' sample to be collected for a study and have signed a consent form saying "yes, you can use this sample for this purpose." Suppose at some point in the future, there is another study, would it be acceptable to you to have the same sample used in the second study?"

$<$ Respondent> "As long as I give consent to it."

$<$ Moderator $>$ "As long as you gave consent to it now or at the time of the second study?"

$<$ Respondent $>$ "I would want to give consent to the other study at a later time, that way I would know what they're using it for. If they're just using it without me knowing, then that's not right."

When it was discussed that requirement for recontact required specimens to be collected in a nonanonymous fashion, perspectives changed.

$<$ Moderator $>$ "If they are able to communicate with you or your loved one in the future, this means that the information provided is no longer anonymous. So they may contact you or your loved one and say "you provided genetic sample for test A, we would like to use it for test B," would you feel comfortable with that?"

$<$ Respondent> "I would want anonymity. Therefore, the consent should tell you that the sample will be preserved, it will be in a database, and it might be used in another study that is strictly controlled. It should specifically say it's not gonna be available for cloning or it's not gonna be for sale. I wouldn't care what kind of test they did, as long as they specified they're gonna look for drugs or treatment."

Finally, one respondent discussed that issues pertaining to future use would most appropriately be addressed by the patient, once sufficiently recovered from their illness.

\section{DISCUSSION}

Most SDMs were familiar with genetic concepts through exposure to mass media, online resources, and personal experience. Despite being confronted with a psychologically stressful situation, the majority of SDMs felt that they could provide an informed opinion regarding permitting (or declining) participation of their loved ones in a study involving collection of genetic data. Consistent with prior studies, principal concerns of SDMs were that a genetic sample might be diverted for inappropriate purposes, or would lead to insurance and employment discrimination. ${ }^{14,18,23,27}$ However, in contrast to these previous investigations, was our finding that SDMs perceived the risk associated with gene variation research largely as the risk associated with acquiring the genetic sample (i.e., whether or not sample acquisition involved an invasive procedure), as opposed to characteristics of the sample itself. This altered frame of reference might reflect the acuteness of the illness of their loved ones, as well as the number and nature of medical interventions these individuals had recently undergone.

The tendency for SDMs to focus on the immediate risks and minimize the long-term consequences of genetic data collection appears to differ from the perception of IRB members. Gong et al. ${ }^{10}$ reported that when presented with a hypothetical genetic epidemiology study involving incapacitated patients, most IRB members reported that such a study would represent greater than minimal risk, and $40 \%$ would not allow such a study to proceed in the absence of direct patient benefit. Similarly, in a multicenter genetic epidemiology acute stroke study, Chen et al. ${ }^{11}$ reported that investigators preferentially enrolled less severely affected, cognitively intact participants because IRBs at $40 \%$ of participating institutions would not permit use of SDMs to provide consent in this context. In contrast, many SDMs in our study voiced the opinion that collection of genetic samples without explicit consent would be acceptable, analogous to the creation of data repositories in nonacute settings. ${ }^{37}$ The tendency to downplay long-term risks associated with genetic data collection might underlie our finding that SDMs perceived genetic data and nongenetic data similarly.

A number of studies have examined opinions regarding future use and return of results. ${ }^{14,16,21,22}$ We found that although SDMs expressed the opinion that either they or their loved ones would be interested in learning individual study results, they requested recontact and reconsent to permit future use. When it was discussed that it would be necessary to archive samples in a nonanonymous fashion so as to enable these requests, SDMs opted for anonymity, foregoing the opportunity to receive study results. Likewise, although permissive of future use, SDMs requested that the parameters of such use be well defined.

When directly questioned, respondents stated that they did not distinguish between genetic and nongenetic data with respect to confidentiality concerns. However, the views actually expressed throughout the FG sessions appeared more nuanced. Specifically, concerns regarding potential forensic applications, paternal attribution, insurance, and employment discrimination appeared more specific to genetic data than to 
nongenetic data. Nonetheless, the preference for anonymity by FG participants has implications for research conducted in the critical care environment. In general, anonymous data collection is less informative than data collected in a manner that can be followed longitudinally, or that can be linked among data resources. Although most critical care studies have a limited time horizon (typical end points being $30-\mathrm{d}$ mortality or hospital discharge), the study of many disease processes requires the ability to perform long-term assessment of outcomes (such as persistent disability or quality of life). Requiring anonymity would render the execution of such studies difficult. The extent to which a study using deidentified data would represent a barrier to enrollment, relative to a study in which data were totally anonymous, is unclear.

Functioning as an SDM has the potential to result in significant emotional duress. ${ }^{31}$ Mehta et al..$^{38}$ examined the attitudes of SDMs who had recently been approached regarding clinical study participation. Those agreeing to participate did so out of altruism, to further medical progress, and because they felt that the patient themselves would have agreed to participate. SDMs declining participation did so predominately because of anxiety. Similarly, Menon et al. ${ }^{39}$ found that SDMs for pediatric patients frequently declined study participation because of emotional duress. Although most of the participants in our study reported that such duress would not interfere with their ability to provide appropriate judgment, these findings collectively suggest that anxiety is a common barrier to research participation. Such perspectives underscore the importance of clinical investigators being sensitive to the dynamics and motivations of SDMs in recruitment efforts and support an argument for exempting low-risk studies from the requirement for informed consent. ${ }^{32}$

SDMs execute their duties according to one of several principles. ${ }^{29}$ SDMs may function as stewards for wishes previously stated by the patient. Alternatively, because such wishes may not have been explicitly expressed, SDMs may operate under the principle of "best interests," acting in good faith to determine what would be in the best interest of the patient. Finally, the SDM might supply "substituted judgment" whereby the SDM decides in such a way that patients would have decided for themselves had they not been incapacitated. Although all participants in our study were actively serving as decisional surrogates for critically ill individuals, and attempting to make decisions that the patients themselves would make if they were able, such decisions and attitudes would be expected to be influenced by personal beliefs and biases. Discrepancies comparing the attitudes held by SDMs with those held by patients may partly account for the relatively poor concordance comparing patient and SDM decisions described previously. ${ }^{40}$ One limitation of our study is that we sampled the opinions of SDMs and not those of the patients themselves, and do not know the extent to which the views of the patient and SDM align. For some of the topics explored (return of results, future use), one could argue that the most ethically rigorous approach would be to obtain patient consent once these individuals had recovered from their critical illness. Such a requirement would present a hurdle to critical care research. The most robust data sets are those that are constructed prospectively. This is particularly true in the ICU setting, where phenotypes are complex and there exists an immense amount of clinical information that is difficult to reconstruct retrospectively. The requirement to obtain informed consent directly from patients would risk losing data that were collected from patients who expired or who never sufficiently recovered to provide consent (a potentially informative subpopulation of critically ill individuals). Furthermore, such a requirement would potentially preclude intervention studies incorporating a genetic component, such as those whose principal analysis is based on genotype, or that prospectively assign treatment based on this information.

\section{Conclusion}

Critical illness studies involving collection of genetic data will require large, multi-institutional cohorts to be maximally informative. ${ }^{2}$ There is variability in the protections applied by IRBs to studies that entail collection of genetic data, as well as to investigations enrolling critically ill individuals. ${ }^{10}$ Such variation is problematic insofar as it potentially introduces interinstitutional differences among study participants. ${ }^{10,11}$ For example, arduous or stringent consent processes in place at one institution may represent a potential barrier to enrollment, relative to institutions adopting a minimalist approach. To the extent that IRB policy can be guided by perspectives and preferences of potential study participants and their decisional surrogates, insight gained from opinion research has value for establishing consent guidelines, and may lead to greater consistency in the consent process across institutions. Furthermore, although SDMs appeared to emphasize the short-term risks associated with genetic data collection, these individuals felt capable of providing informed judgment regarding clinical study participation, and tended to view genetic and nongenetic data comparably. These findings suggest that the consent process should not emphasize whether data are genetic or nongenetic but should clearly detail privacy protections, specimen handling, and parameters that guide future use. Such an approach may be instrumental to both allaying concerns related to research participation and enhancing enrollment.

\section{ACKNOWLEDGMENTS}

The authors are indebted to the nurses, physicians, patients, family members, and personnel of the medical and surgical ICUs of Barnes Jewish Hospital and Parkland Hospital, whose generous cooperation and support made this study possible. This study was supported by GM080591. Portions of this article have been presented in abstract form at the 138th (6th-10th November 2010, Denver, CO) and 139th (29th October-2nd November 2011, Washington, DC) Annual Meetings of the American Public Health Association and the 40th Critical Care Congress (15th-19th January 2011, San Diego, CA) (Critical Care Medicine, 2010, vol. 38, no. 12 (suppl.)).

\section{DISCLOSURE}

The authors declare no conflict of interest. 


\section{REFERENCES}

1. Collins FS. Shattuck lecture-medical and societal consequences of the Human Genome Project. N Eng/ J Med 1999;341:28-37.

2. Freeman $B D$, Kennedy CR, Frankel HL. Ethical considerations in the collection of genetic data from critically ill patients - what do published studies reveal about potential directions in empirical research? Pharmacogenomics Research 2010;10:77-85.

3. Wadman M. James Watson's genome sequenced at high speed. Nature 2008:452:788

4. Naik G. Gene maps are no cure-all: study warns that DNA scanning to predict disease can mislead; "not a crystal ball". Wall Street Journal 3 April 2012.

5. Espeland MA, Dotson K, Jaramillo SA, et al. Consent for genetics studies among clinical trial participants: findings from Action for Health in Diabetes (Look AHEAD). Clinical Trials 2006;3:456.

6. Ad Hoc Statement Committee of the American Thoracic Society. The ethical conduct of clinical research involving critically ill patients in the United States and Canada. Am J Respir Crit Care Med 2004;170:1375-1384.

7. Luce JM. Research ethics and consent in the intensive care unit. Curr Opin Crit Care 2003;9:540-544

8. Luce JM. Is the concept of informed consent applicable to clinical research involving critically ill patients? Crit Care Med 2003;31(3 Suppl):S153-S160.

9. Silverman HJ, Luce JM, Lanken PN, et al.; NHLBI Acute Respiratory Distress Syndrome Clinical Trials Network (ARDSNet). Recommendations for informed consent forms for critical care clinical trials. Crit Care Med 2005:33:867-882

10. Gong MN, Winkel G, Rhodes R, Richardson LD, Silverstein JH. Surrogate consent for research involving adults with impaired decision making: survey of Institutional Review Board practices. Crit Care Med 2010;38: 2146-2154.

11. Chen DT, Meschia JF, Brott TG, Brown RD, Worrall BB; SWISS investigators. Stroke genetic research and adults with impaired decision-making capacity. A survey of IRB and Investigator Practices. Stroke 2008;39:2735.

12. Bates BR, Lynch JA, Bevan JL, Condit CM. Warranted concerns, warranted outlooks: a focus group study of public understandings of genetic research. Soc Sci Med 2005;60:331-344.

13. Diaz VA, Mainous AG $3^{\text {rd }}$, McCall AA, Geesey ME. Factors affecting research participation in African American college students. Fam Med 2008;40: 46-51.

14. Godard B, Marshall J, Laberge C. Community engagement in genetic research: results of the first public consultation for the Quebec CARTaGENE project. Community Genet 2006;10:147-158.

15. Hallowell N, Cooke S, Crawford G, Parker M, Lucassen A. Healthcare professionals' and researchers' understanding of cancer genetics activities: a qualitative interview study. J Med Ethics 2009;35:113-119.

16. Hoeyer K, Olofsson BO, Mjorndal T, Lynoe N. Informed consent and biobanks: a population-based study of attitudes towards tissue donation for genetic research. Scand J Public Health 2004;32:229.

17. Hoop JG, Roberts LW, Hammond KA. Genetic testing of stored biological samples: views of 570 U.S. workers. Genet Test Mol Biomarkers 2009;13:331-337

18. Hull SC, Sharp RR, Botkin JR, et al. Patients' views on identifiability of samples and informed consent for genetic research. Am J Bioeth 2008;8:62-70.

19. McQuillan GM, Pan Q, Porter KS. Consent for genetic research in a general population: an update on the National Health and Nutrition Examination Survey experience. Genet Med 2006:8:354-360.

20. Marshall PA, Adebamowo CA, Adeyemo AA, et al. Voluntary participation and informed consent to international genetic research. Am J Public Health 2006;96:1989-1995.
21. Moutel G, Duchange N, Raffi F, et al.; APROCO-COPILOTE Study Group. Communication of pharmacogenetic research results to HIV-infected treated patients: standpoints of professionals and patients. Eur J Hum Genet 2005;13:1055-1062.

22. Murphy J, Scott J, Kaufman D, Geller G, LeRoy L, Hudson K. Public expectations for return of results from large-cohort genetic research. $\mathrm{Am} J$ Bioeth 2008;8:36-43.

23. Murphy E, Thompson A. An exploration of attitudes among black Americans towards psychiatric genetic research. Psychiatry 2009;72:177-194.

24. Ormondroyd E, Moynihan C, Watson M, et al. Disclosure of genetics research results after the death of the patient participant: a qualitative study of the impact on relatives. J Genet Couns 2007;16:527-538.

25. Schulz A, Caldwell C, Foster S. "What are they going to do with the information?" Latino/Latina and African American perspectives on the Human Genome Project. Health Educ Behav 2003;30:151-169.

26. Vermeulen $E$, Schmidt MK, Aaronson NK, Kuenen M, van Leeuwen FE. Obtaining "fresh" consent for genetic research with biological samples archived 10 years ago. Eur J Cancer 2009:45:1168-1174.

27. van der Vorm A, Rikkert MO, Vernooij-Dassen M, Dekkers W; EDCON Panel. Genetic research into Alzheimer's disease: a European focus group study on ethical issues. Int J Geriatr Psychiatry 2008:23:11-15.

28. Freeman BD, Kennedy CR, Coopersmith CM, Zehnbauer BA, Buchman TG Genetic research and testing in critical care: surrogates' perspective. Crit Care Med 2006:34:986-994.

29. Lavery JV, Slutsky AS. Substitute decisions about genetic testing in critical care research: a glimpse behind the curtain. Crit Care Med 2006;34:12571259.

30. Freeman BD, Kennedy CR, Bolcic-Jankovic D, et al. Considerations in the construction of an instrument to assess attitudes regarding critical illness gene variation research. J Empir Res Hum Res Ethics 2012;7:58-70.

31. Pochard F, Azoulay E, Chevret S, et al. Symptoms of anxiety and depression in family members of intensive care unit patients: ethical hypothesis regarding decision-making capacity. Crit Care Med 2001;29: 1983-1987.

32. Barrett KA, Scales DC. Considering the vulnerabilities of surrogate decisionmakers when obtaining consent for critical care research. Intensive Care Med 2012:38:4-6.

33. Green MJ, Botkin JR. "Genetic exceptionalism" in medicine: clarifying the differences between genetic and nongenetic tests. Ann Intern Med 2003; 138:571-575.

34. Trinidad SB, Fullerton SM, Ludman EJ, Jarvik GP, Larson EB, Burke W. Research ethics. Research practice and participant preferences: the growing gulf. Science 2011;331:287-288.

35. Iverson E, Celious A, Kennedy CR, et al. Real-time perspectives of surrogate decision makers regarding critical illness research-findings of focus group participants. Chest 2012, in press.

36. Glaser BG. The Discovery of Grounded Theory: Strategies for Qualitative Research. Weidenfeld and Nicholson: Chicago, IL, 1968.

37. Pulley J, Clayton E, Bernard GR, Roden DM, Masys DR. Principles of human subjects protections applied in an opt-out, de-identified biobank. Clin Trans Sci 2010;3:42-48.

38. Mehta S, Quittnat Pelletier F, Brown M, et al. Why substitute decision makers provide or decline consent for ICU research studies: a questionnaire study. Intensive Care Med 2012;38:4754-4754.

39. Menon K, Ward RE, Gaboury I, et al. Factors affecting consent in pediatric critical care research. Intensive Care Med 2012;38:153-159.

40. Coppolino M, Ackerson L. Do surrogate decision makers provide accurate consent for intensive care research? Chest 2001;119:603-612. 